Check for updates

Cite this: RSC Adv., 2018, 8, 20549

\title{
Biomimetic enzyme-linked immunoassay based on a molecularly imprinted 96-well plate for the determination of triazophos residues in real samples
}

\author{
Sihui Hong, ${ }^{a}$ Yongxin She, (DD *a Xiaolin Cao, ${ }^{a}$ Miao Wang, (D) a Chao Zhang, ${ }^{a}$ \\ Lufei Zheng, ${ }^{a}$ Shanshan Wang, ${ }^{a}$ Xingbin Ma, ${ }^{b}$ Hua Shao, ${ }^{a}$ Maojun Jin, ${ }^{a}$ Fen $\mathrm{Jin}^{\mathrm{a}}$ \\ and Jing Wang*a
}

In this study, a direct competitive biomimetic enzyme-linked immune-sorbent assay (BELISA) based on a molecularly imprinted nanomembrane as an artificial antibody was developed for the determination of triazophos in real samples. The imprinted film was directly synthesized on the well surface of a 96-well plate using a dummy molecular template under the conditions of thermal polymerization. The developed BELISA using a hapten of triazophos as an enzyme-labeled probe is much more sensitive, simple, quick, steady and inexpensive than the other instrumental and immuno assay methods. Under optimal conditions, the linear range of the method was $0.001-10000 \mu \mathrm{g} \mathrm{L}^{-1}$ with a good regression coefficient of 0.977 . The sensitivity $\left(\mathrm{IC}_{50}\right)$ and the limit of detection (LOD) of BELISA were $428 \mu \mathrm{g} \mathrm{L}^{-1}$ and $0.001 \mu \mathrm{g} \mathrm{L}^{-1}$, respectively. This method was performed to detect triazophos in cabbage and apple samples, and showed excellent recovery and relative standard deviations (RSDs) ranging from 70.5 to $119.8 \%$ and from 5.2 to $19.7 \%$, respectively. The results correlated well with those obtained using high performance liquid chromatography-tandem mass spectrometry.

Received 24th April 2018

Accepted 23rd May 2018

DOI: 10.1039/c8ra03531h

rsc.li/rsc-advances

\section{Introduction}

Triazophos is a moderately toxic, broad-spectrum organophosphate pesticide that has been widely used in agriculture to control insect pests. ${ }^{1}$ Due to its relative stability, widespread use and long half-life, residues of triazophos in agricultural products and the natural environment may result in potential hazards to human health and animals. ${ }^{2}$ Since the end of 2016, triazophos has been banned for use on vegetables by the Chinese Ministry of Agriculture, but the illegal use of triazophos for vegetables and fruits still happens now and then. Therefore, it is important to develop a highly sensitive and selective method for the detection of triazophos residues in agricultural and environmental samples. ${ }^{3}$

So far, various analytical methods for the effective monitoring of triazophos have been developed, such as gas chromatography (GC), ${ }^{4}$ gas chromatography with mass spectrometry (GC/MS), ${ }^{5}$ liquid chromatography with mass spectrometry (LC/ MS), ${ }^{6}$ and enzyme/antibody-based immunoassays. ${ }^{7}$ The LC, GC

${ }^{a}$ Institute of Quality Standard and Testing Technology for Agro-Products, Chinese Academy of Agricultural Science, Key Laboratory of Agro-Products Quality and Safety of Chinese Ministry of Agriculture, Beijing 100081, P. R. China. E-mail: 0891syx@ 163.com; Fax: +86 010 82106567; Tel: +8610 82106513

${ }^{b}$ Institute of Veterinary and Animal Husbandry, Tibet Academy of Agricultural and Animal Husbandry Sciences, Lhasa 850006, P. R. China method, or that coupled with MS, are common methods with high sensitivity and accuracy and good reproducibility, but the instrument size, the high costs, the need for skilled technicians and the lengthy analysis time limit their wide utilization and direct field detection. ${ }^{8}$ Immunoassays, especially enzyme-linked immunosorbent assays (ELISA), are also sensitive and selective and can run many analyses simultaneously without the need for skilled technicians. ${ }^{9}$ However, they have many drawbacks, including the high commercial cost, reagent stability, difficulties associated with antibody production and the use of laboratory animals. ${ }^{9}$ Thus, it is necessary to develop a sensitive, simple, quick, stable and inexpensive method to monitor the residual quantity of triazophos in real samples.

Molecular imprinting is a method used to create selective recognition sites in synthetic polymers. ${ }^{10}$ The molecularly imprinted polymers (MIPs) have properties such as predictable specific recognition, easy preparation, low cost, high mechanical/chemical stability and good rigidity. ${ }^{11,12}$ Due to the above advantages, MIPs are described as artificial antibodies that can replace antibodies and have been applied in some biomimetic enzyme-linked immunosorbent assay (BELISA) methods. ${ }^{13-16}$

Usually, it is difficult to fully eliminate the molecular template from MIPs, so leakage of the molecular template during the use of MIPs resulted in inaccuracy of the analytical 
results. Triadimefon, whose structure is similar to triazophos but has a lower toxicity, would be available as a dummy template molecule to further reduce the number of false positive results. In this study, we chose triadimefon as a molecular template to synthesize MIPs as a recognition unit on a 96-well plate and also constructed a labelling probe using a hapten of triazophos labelled as horseradish peroxidase. Subsequently, a sensitive, simple, quick, and directly competitive BELISA method for the detection of triazophos was developed, using the imprinted film as an artificial antibody. The parameters affecting the performance of the BELISA were optimized in detail. This developed method was applied to the analysis of triazophos in cabbage and apple samples, and the results were validated by HPLC-MS/MS.

\section{Materials and methods}

\subsection{Reagents and chemicals}

Triazophos was obtained from Aladdin Chemistry Co. Ltd. (Shanghai, China). Parathion, chlorpyrifos, triadimefon, and methomyl were purchased from Dr Ehrenstorfer Gmbh (Augsburg, Germany). Methacrylic acid (MAA) and trimethylolpropane trimethacrylate (TRIM) were purchased from Alfa Aesar (Massachusetts, USA). The free radical initiator 2,2-azobisisobutyronitrile (AIBN) was purchased from the no. 4 reagent and H. V. Chemical Co., Ltd. (Shanghai, China). The triazophos hapten was gifted by the Institute of Pesticide and Environmental Toxicology, Zhejiang University, China. Horseradish peroxidase (HRP) was bought from Solarbio (Beijing, China). $N$ Dicyclohexylcarbodiimide (DCC), and $N$-hydroxysuccinate (NHS) were purchased from Sigma-Aldrich (St. Louis, MO, USA). The 3,3',5,5'-tetramethylbenzidine (TMB) ELISA substrate was purchased from TransGen Biotech Co., Ltd (Beijing, China). All the other chemicals and organic solvents used in this study were of the highest available purity and at least of analytical grade. Maxisorp polystyrene 96-well plates were obtained from Corning, Inc. (Corning, NY, USA). Ultrapure water $(\geq 18.2 \mathrm{M} \Omega$ $\mathrm{cm}$ ) produced by a Milli-Q water purification system (Millipore, Bedford, MA, USA) was used in all experimental works.

An assay buffer consisting of $10 \mathrm{mM}$ PBS (pH 7.4), a washing buffer (PBST) (consisting of $10 \mathrm{mM}$ PBS (pH 7.4) and $0.05 \%$ Tween 20) and a stopping solution ( $\left.2 \mathrm{M} \mathrm{H}_{2} \mathrm{SO}_{4}\right)$ were used in this study.

\subsection{Preparation of the molecularly imprinted film on the surface of the 96-well plate}

The imprinted film was directly polymerized in the wells of the 96-well plate according to the following procedure: $588 \mathrm{mg}$ (2 $\mathrm{mmol}$ ) of triadimefon was dissolved in $20 \mathrm{~mL}$ of acetonitrile in a $150 \mathrm{~mL}$ flask, and then mixed with $1020 \mu \mathrm{L}$ (12 mmol) of MAA. After magnetically stirring for $30 \mathrm{~min}$ at room temperature, $1916 \mu \mathrm{L}(6 \mathrm{mmol})$ of TRIM and $50 \mathrm{mg}$ of AIBN were added in turn. The mixture was continuously stirred for $60 \mathrm{~min}$. Then, $100 \mu \mathrm{L}$ of the mixture was placed in each well of the 96 -well plate and the reaction was carried out under vacuum for $24 \mathrm{~h}$ at $37^{\circ} \mathrm{C}$ by a vacuum oven.
After polymerization was complete, the plate was washed three times with DDW and methanol, respectively, to remove any unreacted solution. The imprinted film was first extracted with methanol/acetic acid ( $7: 1, \mathrm{v} / \mathrm{v})$ followed by methanol for $4 \mathrm{~h}$ using an ultrasonic cleaner until no triadimefon was detected in the eluent by HPLC. For comparison, a nonimprinted film was synthesized by the same procedure as above without the addition of triadimefon. The process is shown in Fig. 1(a).

\subsection{Characterization of the imprinted film}

The morphological characterization was analyzed using a fieldemission scanning electron microscope (JSM-6300, Japan). The bonding effects in the polymers were evaluated by Fourier transform infrared (FT-IR) spectra (4000-400 $\mathrm{cm}^{-1}$ ) with a Philips PU9800 spectrometer (Philips Analytical, Cambridge, UK).

\subsection{Adsorption dynamic experiments}

To evaluate the binding kinetics of the imprinted film, $200 \mu \mathrm{L}$ of PBS solution containing triazophos (at $100 \mathrm{mg} \mathrm{L}^{-1}$ ) was added to the 96-well plate. After shaking for 5, 10, 20, 30, 40, 50, 60, 70, and $80 \mathrm{~min}$ at room temperature, the supernatants were analyzed by HPLC.

To measure the adsorption capacity, the imprinted or nonimprinted film was equilibrated with $200 \mu \mathrm{L}$ of PBS solution containing triazophos at various concentrations (10$100 \mathrm{mg} \mathrm{L}^{-1}$ ). After shaking for $50 \mathrm{~min}$ at room temperature, the supernatants were analyzed by HPLC. The polymer adsorption capacity $\left(Q, \mu \mathrm{g}\right.$ per well) was calculated using eqn (1). ${ }^{17}$

$$
Q=\left(C_{0}-C_{1}\right) V \times 1000
$$

where $C_{0}$ and $C_{1}\left(\mathrm{mg} \mathrm{\textrm {L } ^ { - 1 }}\right)$ are the initial and equilibrium concentrations of triazophos, respectively; $V(\mathrm{~L})$ is the volume of adsorption solution in each well. The imprinting factor $(\alpha)$ was calculated using eqn (2). ${ }^{18}$

$$
\alpha=\frac{Q_{\mathrm{MIP}}}{Q_{\mathrm{NIP}}}
$$

where $Q_{\text {MIP }}$ and $Q_{\text {NIP }}$ are the adsorption capacity of the MIP and NIP films, respectively.

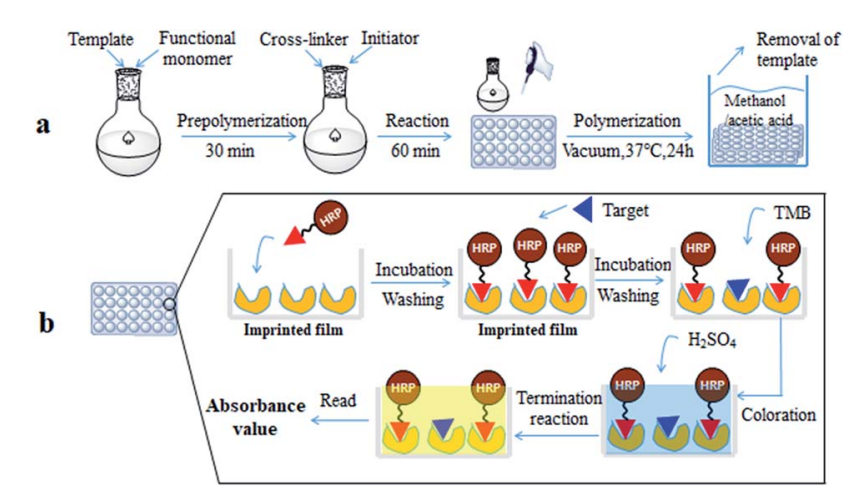

Fig. 1 Preparation of the molecularly imprinted film on the surface of the 96-well plate (a); direct competitive BELISA procedure (b). 


\subsection{Synthesis of the enzyme-labeled probe of triazophos}

The hapten of triazophos was supplied by Zhejiang University. The synthesis of the enzyme-labeled probe of triazophos was based on the literature ${ }^{19}$ with certain modifications. First, $18.85 \mathrm{mg}(0.05 \mathrm{mmol})$ of the hapten of triazophos was dissolved in $0.5 \mathrm{~mL}$ of DMF as solution (a). Next, $17.25 \mathrm{mg}(0.15$ $\mathrm{mmol}$ ) of NHS was added to solution (a) and stirred for $15 \mathrm{~min}$ as solution (b). Then, $15.46 \mathrm{mg}(0.075 \mathrm{mmol})$ of DCC was dissolved in $0.5 \mathrm{~mL}$ of DMF, and added to solution (b) drop by drop. The mixture was magnetically stirred at room temperature over-night. After centrifugation (4000 rpm, $10 \mathrm{~min}$ ), the supernatant was preserved as solution (c). Solution (d) was prepared by adding $40 \mathrm{mg}$ of HRP to $4 \mathrm{~mL}$ of $10 \mathrm{mmol} \mathrm{L}^{-1} \mathrm{CBS}$ solution. After that, $400 \mu \mathrm{L}$ of solution (c) was added dropwise to solution (d), and the mixture was stirred for $4 \mathrm{~h}$ at room temperature. The enzyme conjugated solution was then dialyzed against PBS $\left(10 \mathrm{mmol} \mathrm{L}^{-1}\right)$. Finally, the volume of the conjugated enzyme was accurately calculated, and the same volume of glycerine was added to the solution, and stored at $-20{ }^{\circ} \mathrm{C}$ before use.

\subsection{Direct competitive BELISA procedure}

The direct competitive BELISA procedure was carried out using the imprinted film on the 96-well plate as a biomimetic antibody. First, the plate was washed three times with PBST (PBS with $0.05 \%$ (v/v) Tween 20). Then, $100 \mu \mathrm{L}$ of PBS was added to the blank, $100 \mu \mathrm{L}$ of the enzyme labelled probe was added to all wells except for the blank, and the 96-well plate was shaken for $30 \mathrm{~min}$ at room temperature. After that, the plate was washed with PBST ten times. Next, $5 \%$ methanol PBS (v/v) was added to the blank $(100 \mu \mathrm{L})$ and the control $(100 \mu \mathrm{L})$ wells, $100 \mu \mathrm{L}$ of the standard solution or sample extracts were added to the allocated wells, and the 96-well plate was shaken for $1 \mathrm{~h}$ at room temperature. Following washing with PBST ten times, $100 \mu \mathrm{L}$ of the TMB ELISA substrate solution was added to each well. After coloration for $15 \mathrm{~min}$ at $37{ }^{\circ} \mathrm{C}$, the reaction was terminated by adding $50 \mu \mathrm{L}$ of $\mathrm{H}_{2} \mathrm{SO}_{4}\left(2 \mathrm{~mol} \mathrm{~L}{ }^{-1}\right)$ per well. The whole mixture in each well was transferred to another 96-well plate, and the UV absorbance was recorded using a Labsystems 96-well plate reader (TECAN, Switzerland) in dual-wavelength mode (450-650 $\mathrm{nm}$ ). The process is shown in Fig. 1(b). The inhibition rate (IC\%) of triazophos at various concentrations was calculated using eqn (3).

$$
\mathrm{IC}(\%)=\left[1-\frac{A_{\mathrm{s}}-A_{\mathrm{o}}}{A_{\mathrm{c}}-A_{\mathrm{o}}}\right] \times 100
$$

where IC\% is the inhibition rate to the target-MIP binding reaction; $A_{\mathrm{S}}$ is the average absorbance value of the standard solution or sample; $A_{\mathrm{C}}$ is the average absorbance value obtained by the wells without the addition of the standard solution; $A_{0}$ is the average absorbance value obtained by the wells without the addition of the standard solution and the enzyme labelled probe. Finally, the imprinted film on the plate was cleaned again using methanol/acetic acid ( $7: 1)$, followed by methanol, for the next BELISA procedure.

\subsection{Selectivity of BELISA}

Cross-reactivity (CR) studies were carried out by measuring the competitive curves and selectivity properties of the structural analogs and several chemically related compounds. In this paper, parathion, chlorpyrifos, triadimefon, and methomyl were selected to evaluate the selectivity of BELISA. The CR was calculated using eqn (4).

$$
\begin{aligned}
\mathrm{CR} \%= & \left\{\mathrm{IC}_{50}(\text { triazophos }) / \mathrm{IC}_{50}(\text { cross-reacting compound })\right\} \\
& \times 100
\end{aligned}
$$

\subsection{Sample preparation}

To evaluate the performance of BELISA, some cabbage and apple samples were purchased from a local market to carry out the spiked recovery studies. Before spiking, the samples were determined to be free of triazophos by HPLC-MS/MS. The pretreatment of the cabbage and apple samples was based on the literature ${ }^{20}$ with slight modifications. Briefly, $10 \mathrm{~g}$ samples were weighed into $50 \mathrm{~mL}$ centrifuge tubes, which were spiked with triazophos at three levels, 10, 50, and $500 \mu \mathrm{g} \mathrm{kg}^{-1}$. Then $10 \mathrm{~mL}$ acetonitrile was added and shaken vigorously by a vortex for $2 \mathrm{~min}$. After that, $4 \mathrm{~g} \mathrm{MgSO}$ and $1 \mathrm{~g} \mathrm{NaCl}$ were added and shaken for $2 \mathrm{~min}$, and then centrifuged for $5 \mathrm{~min}$ at $5000 \mathrm{rpm}$. $2 \mathrm{~mL}$ of supernatant was transferred to a $10 \mathrm{~mL}$ centrifuge tube

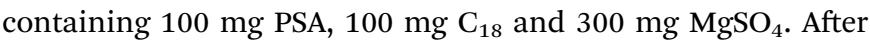
being shaken for $2 \mathrm{~min}$, the mixture was centrifuged at $10000 \mathrm{rpm}$ for $5 \mathrm{~min}$. Half of the supernatant was used for HPLC-MS/MS detection and the other was dried using nitrogen. Finally, the residue was dissolved in 5\% methanol-PBS (diluted 20 times) and analyzed by the BELISA method.

\subsection{HPLC analysis and HPLC-MS/MS validation}

The HPLC analysis was carried out using a Waters 2695 Alliance HPLC system (Waters Corporation, Milford, MA, USA) equipped with a DAD detector at $246 \mathrm{~nm}$ for triazophos and $224 \mathrm{~nm}$ for triadimefon. Separation was carried out using a Waters XBridge $\mathrm{C}_{18}$ column $(4.6 \times 250 \mathrm{~mm}$, particle size $5 \mu \mathrm{m})$. The column temperature was $25{ }^{\circ} \mathrm{C}$. The mobile phase was water-acetonitrile $(20: 80, \mathrm{v} / \mathrm{v})$ for triazophos and triadimefon in isocratic mode at a flow rate of $0.8 \mathrm{~mL} \mathrm{~min}{ }^{-1}$, and the injection volume was $10 \mu \mathrm{L}$.

HPLC-MS/MS analysis was carried out using an Agilent Series 1200 liquid chromatograph (Agilent Technologies, Palo Alto, CA, USA), with an API2000 triple quadrupole mass spectrometer equipped with a turbo-ion spray ionization source (Applied Biosystem/MDS Sciex, Foster City, CA, USA). The MS was operated in the multiple reaction monitoring (MRM) mode using positive ionization. Triazophos was identified in the positive scan mode at $m / z 314.1$, and the quantifier ion $(\mathrm{m} / \mathrm{z})$ and qualifier ion $(\mathrm{m} / \mathrm{z})$ were 162.2 and 286.0, whose declustering potential $(\mathrm{DP}, \mathrm{v} / \mathrm{v})$ values were both 52.60 , while the collision energy (CE, v/v) values were 24.96 and 28.36 , respectively. The ion source temperature was $450{ }^{\circ} \mathrm{C}$ and the gas pressure of the collision activated dissociation (CAD), the curtain gas (CUR), ion source gas 1 (GS1), and ion source gas 2 (GS2) were 10, 50, 
35 , and 35 psi, respectively. The mobile phase consisted of $0.1 \%$ formic acid and $5 \mathrm{mM}$ ammonium acetate in ultra-pure water (A)-acetonitrile (B) (40:60 v/v), using an XBridge $\mathrm{C}_{18}$ column $(150 \times 2.1 \mathrm{~mm}, 5 \mu \mathrm{m}$, Waters, Ireland $)$ at a flow rate of 250 $\mu \mathrm{L} \min ^{-1}$. The injection volume was set to $5 \mu \mathrm{L}$.

\section{Results and discussion}

\subsection{Optimizing the preparation of the MIP films}

The synthesis conditions, including the mass ratio of template to functional monomer and the mass ratio of template to crosslinker, were optimized based on the adsorption capacity of the MIP/NIP films for triazophos. The MIP films were synthesized at different mass ratios $(1: 2,1: 3,1: 4,1: 5$, and $1: 6)$ of template to functional monomer and different mass ratios ( $1: 1,1: 2,1: 3,1: 4$, and $1: 5)$ of template to cross-linker. The results in Fig. 2(a) and (b) show that the imprinting factor reaches a maximal value of 1.17 and 1.51 at the mass ratio $(1: 6)$ of template to functional monomer and the mass ratio $(1: 3)$ of template to cross-linker, respectively.

\subsection{FT-IR spectra and SEM of the imprinted film}

The FT-IR spectra of triadimefon (a), the imprinted film before extraction of the template (b), the imprinted film after extraction of the template (c) and the non-imprinted film (d) are shown in Fig. 3. In both spectra (a) and (b), there are peaks $670 \mathrm{~cm}^{-1}$ and $649 \mathrm{~cm}^{-1}$, which are characteristic of the benzene
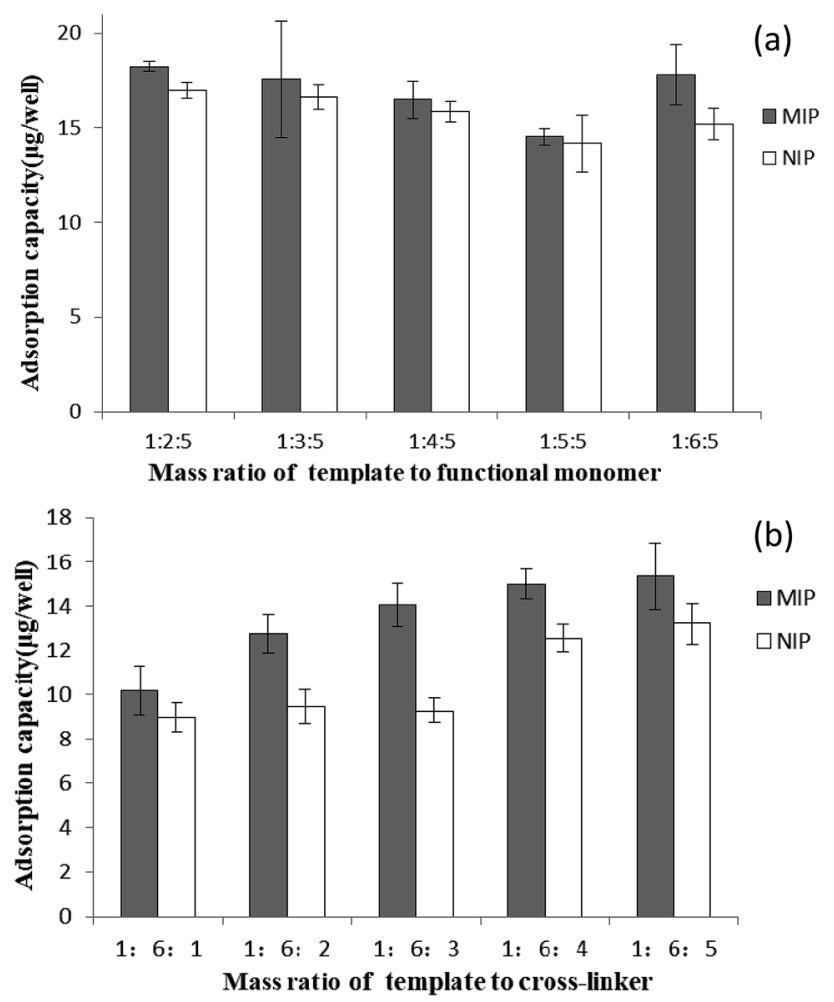

Fig. 2 Adsorption capacities of the MIP and NIP films synthesized at different mass ratios of template to functional monomer (a); and different mass ratios of template to cross-linker (b).

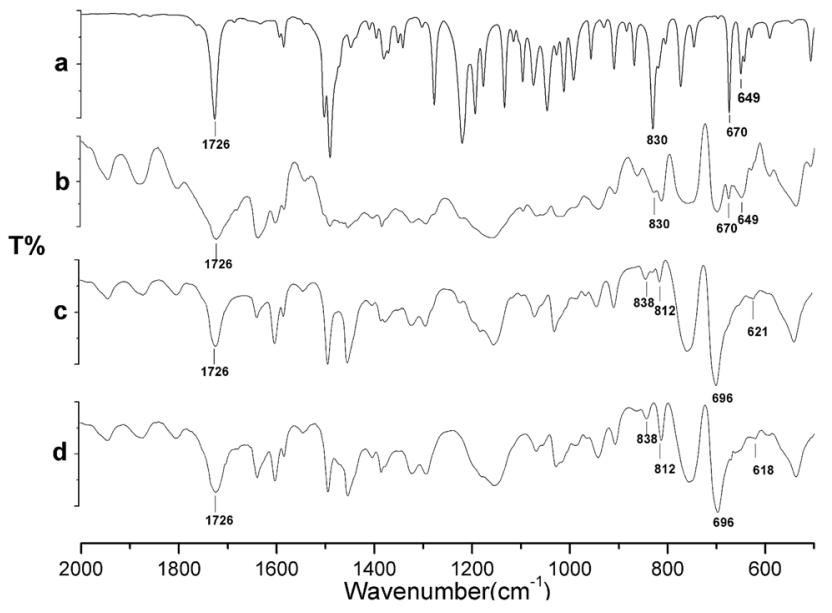

Fig. 3 FT-IR spectra of triadimefon (a), the imprinted film before extraction (b), the imprinted film after extraction (c) and the nonimprinted film (d).

ring and the chlorine on the benzene ring in triadimefon, which are not found in spectra (c) and (d). Spectrum (c) is similar to spectrum (d). These results indicate that the template molecule was anchored on the imprinted polymer framework successfully and could be completely removed after extraction. Meanwhile, after the extraction of the template, the peak in the MIP spectrum near $830 \mathrm{~cm}^{-1}$ disappeared and the peak near $1726 \mathrm{~cm}^{-1}$ was weakened, as shown in Fig. 3(c), and the same peaks are present for the NIP.

In order to investigate the structure, the imprinted film before extraction (a), the imprinted film after extraction (b) and the non-imprinted film (c) were visualized using SEM (Fig. 4). The SEM images revealed that the surface of the imprinted film was markedly different from that after the removal of the template from the MIP. The surface of the imprinted film was much rougher than the non-imprinted film, which manifested that the specific binding sites could be formed in the imprinted film.

\subsection{Adsorption properties of the imprinted film}

The adsorption dynamics of triazophos on the imprinted film was investigated in $100 \mathrm{mg} \mathrm{L}^{-1}$ of triazophos solution. As shown in Fig. 5, the imprinted film had a fast adsorption capacity, $74.1 \%$ of binding was achieved within a short period of $10 \mathrm{~min}$, and an equilibrium value was reached at $50 \mathrm{~min}$.

Besides this, the isothermal adsorption curves of the imprinted and the non-imprinted films were obtained using

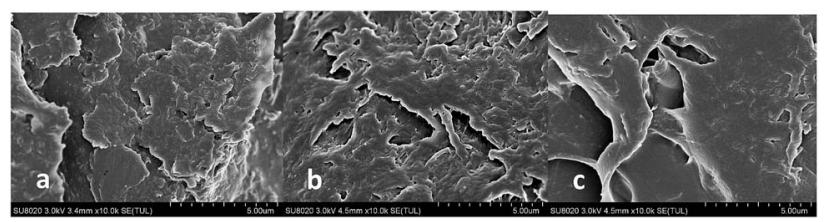

Fig. 4 SEM images of the imprinted film before extraction (a), the imprinted film after extraction (b) and the non-imprinted film (c). 


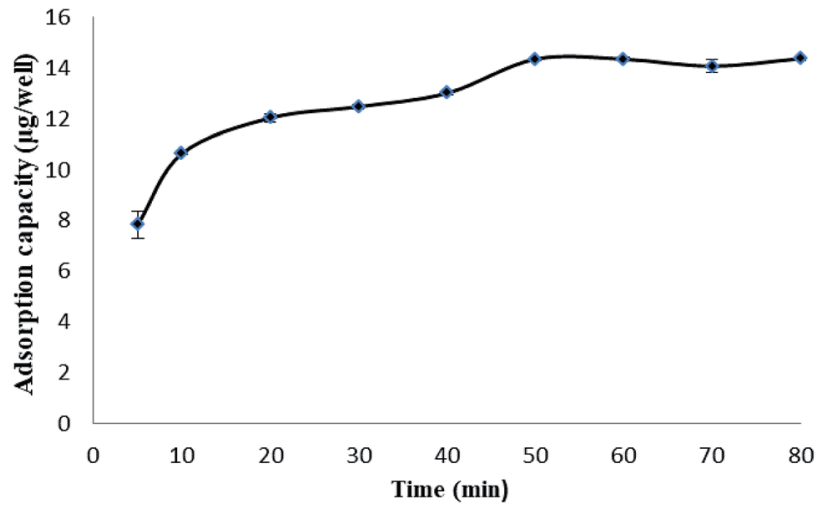

Fig. 5 Adsorption kinetic curve of the MIP films.

a series of triazophos-PBS solutions $\left(10-100 \mathrm{mg} \mathrm{L}^{-1}\right)$, and are plotted in Fig. 6. The results indicate that the adsorption capacities of the imprinted and the non-imprinted films increased with the initial concentration, and the imprinted films exhibited higher adsorption capacities than the nonimprinted films at the same triazophos level.

\subsection{Optimization of the BELISA conditions}

In order to achieve optimal sensitivity and precision for the BELISA method, the parameters, including the order of adding the enzyme labelled probe and the standard solution, the number of times the plate was washed, the enzyme labelled probe concentration, the preparation of the solvent and the thickness of the imprinted film, were optimized in detail.

The order of adding the enzyme labelled probe and the standard solution could affect the BELISA. The experimental results show that the competitive effect of the separate addition of the enzyme labelled probe and the standard solution was much better than the simultaneous addition of both of them. The number of times the plate was washed could affect the sensitivity of the method. In order to investigate the influence on the performance of the assay, the number of times the plate was washed $(5,10$, and 15$)$ was tested by comparing the inhibition. Washing ten times had a higher inhibition. Washing more times may reduce nonspecific adsorption to increase the

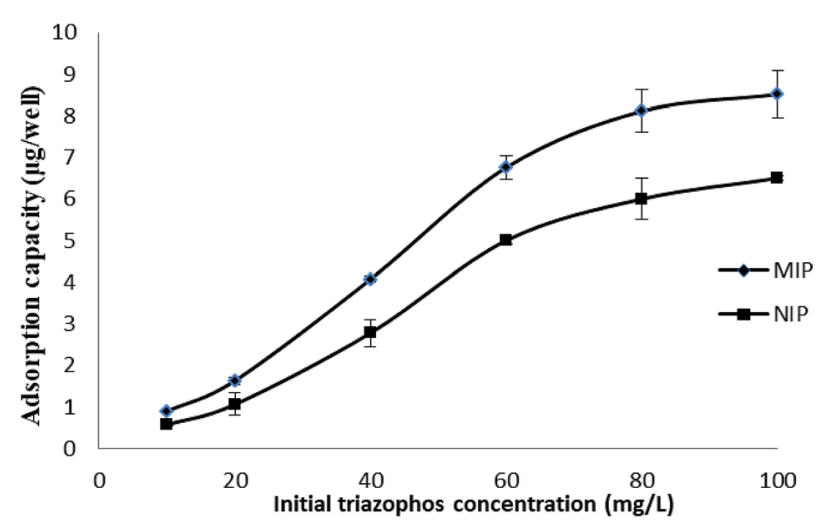

Fig. 6 Adsorption isotherms of the MIP and NIP films. competitive effect, but washing too much may wash away specific adsorption at the same time.

The sensitivity of the BELISA method can be affected by the concentration of the enzyme labelled probe. Different dilution ratios $(1: 100,1: 200,1: 400,1: 600,1: 800$, and $1: 1000)$ of the enzyme labelled probe were used in the BELISA procedure. Fig. 7 shows that the absorbance value decreased with decreasing concentration of the enzyme labelled probe. The concentration of the enzyme labelled probe is usually determined by the titre of enzyme tracers to yield absorbance values ranging from 1 to 1.2. In this study, the enzyme labelled probe was diluted to $1: 600$ before use.

The solvent used for the preparation of the standard solution and the samples could affect the selectivity and the recognition ability of the imprinted films, and the sensitivity of BELISA. In order to investigate the influence on the performance of the assay, methanol solutions at $0,5,10$, and $15 \%$ in PBS were tested by comparing the inhibition and the $\mathrm{IC}_{50}$ value obtained from the standard curves. The results (Fig. 8) indicate that the

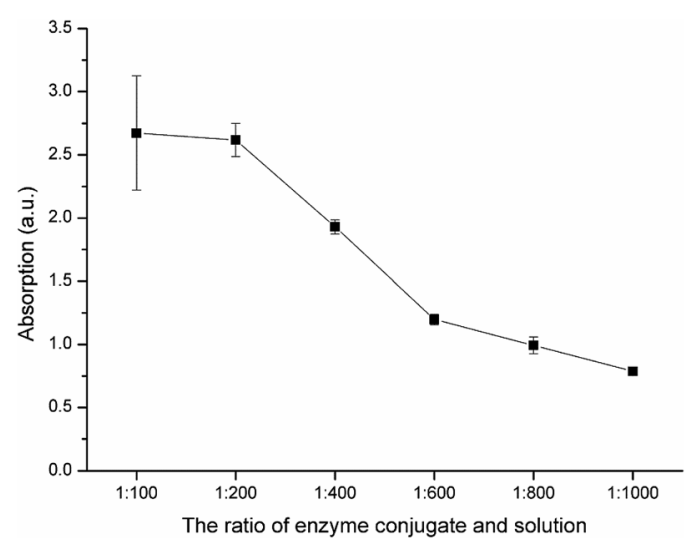

Fig. 7 The absorbance values of the enzyme labelled probe at different dilution ratios.

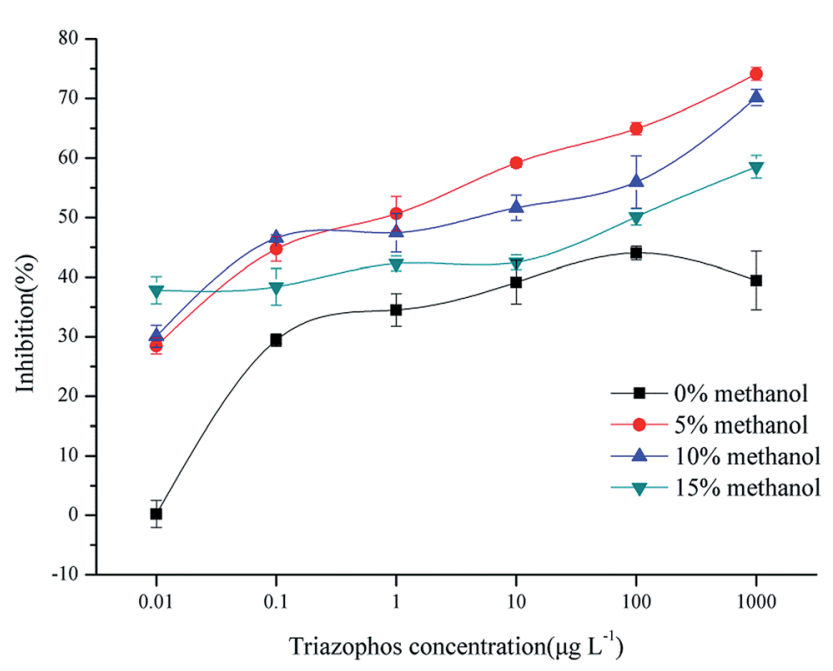

Fig. 8 BELISA standard curves of triazophos using the imprinted film as an artificial antibody in PBS containing different concentrations of methanol. 
inhibition ratio and the sensitivity were the highest in the 5\% methanol PBS system. Therefore, the 5\% methanol PBS solution was chosen as the preparation solvent for the routine analysis of triazophos in real samples.

The thickness of the imprinted film may have a direct effect on the assay sensitivity. In order to investigate the influence, the coating amount of the imprinted film containing different volumes of pre-polymer solution $(50,100$, and $200 \mu \mathrm{L})$ were tested. The results show that the imprinted film could not fully coat on the well surface of the 96-well plate using $50 \mu \mathrm{L}$ of pre-polymer solution, and it was too thick and had a lower sensitivity using $200 \mu \mathrm{L}$. Thus, $100 \mu \mathrm{L}$ of pre-polymer solution was added into each well of the plate to form a thin and uniform imprinted film.

\subsection{Cross-reactivity of the BELISA method}

The cross-reactivity of the BELISA method was evaluated using competitive assays with related compounds. Parathion and chlorpyrifos were selected as organophosphorus pesticides with similar molecular structures to triazophos; triadimefon was selected as a dummy molecular template; methomyl was selected as a non-benzene ring compound. The results are shown in Fig. 9 and Table 1.

Relative to triazophos, the CR of parathion and triadimefon are $7.47 \%$ and $0.25 \%$, respectively. This result could indicate that the imprinted films have specific recognition capability toward compounds with a triazole ring, a benzene ring or a phosphoester bond when selecting triadimefon as the dummy template and the hapten of triazophos as the enzyme labelled probe. However, the CR values for parathion and triadimefon were less than $10 \%$, which indicates that the imprinted films still have high selectivity toward triazophos. Besides this, the CR of chlorpyrifos and methomyl were less than $0.04 \%$, which shows that the imprinted films have low sensitivity toward non phenyl pesticides.

\subsection{Parameters of the BELISA method}

The normalized competition curves obtained using the novel imprinted film and the non-imprinted film as the artificial

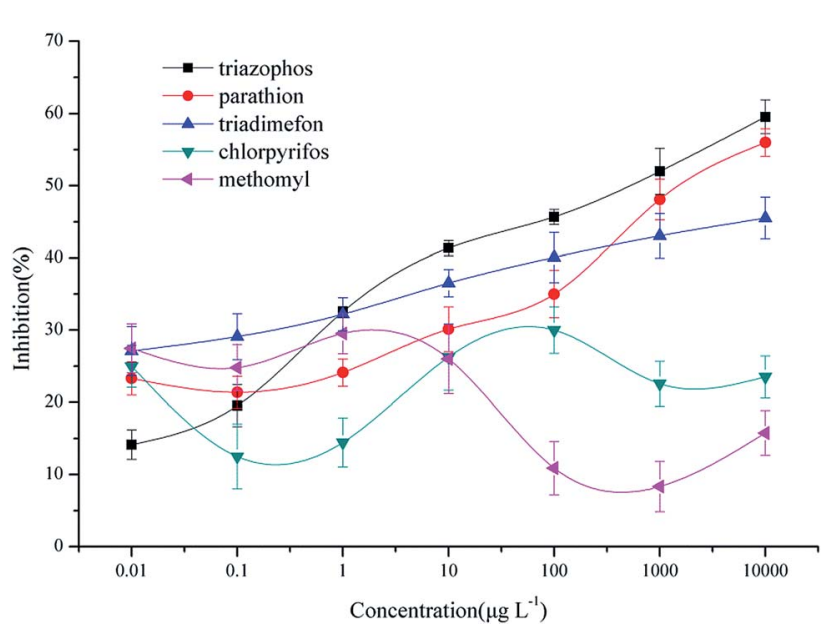

Fig. 9 Standard BELISA competition curves for triazophos and the related compounds.
Table 1 Cross-reactivities (CR\%) between triazophos and the related compounds
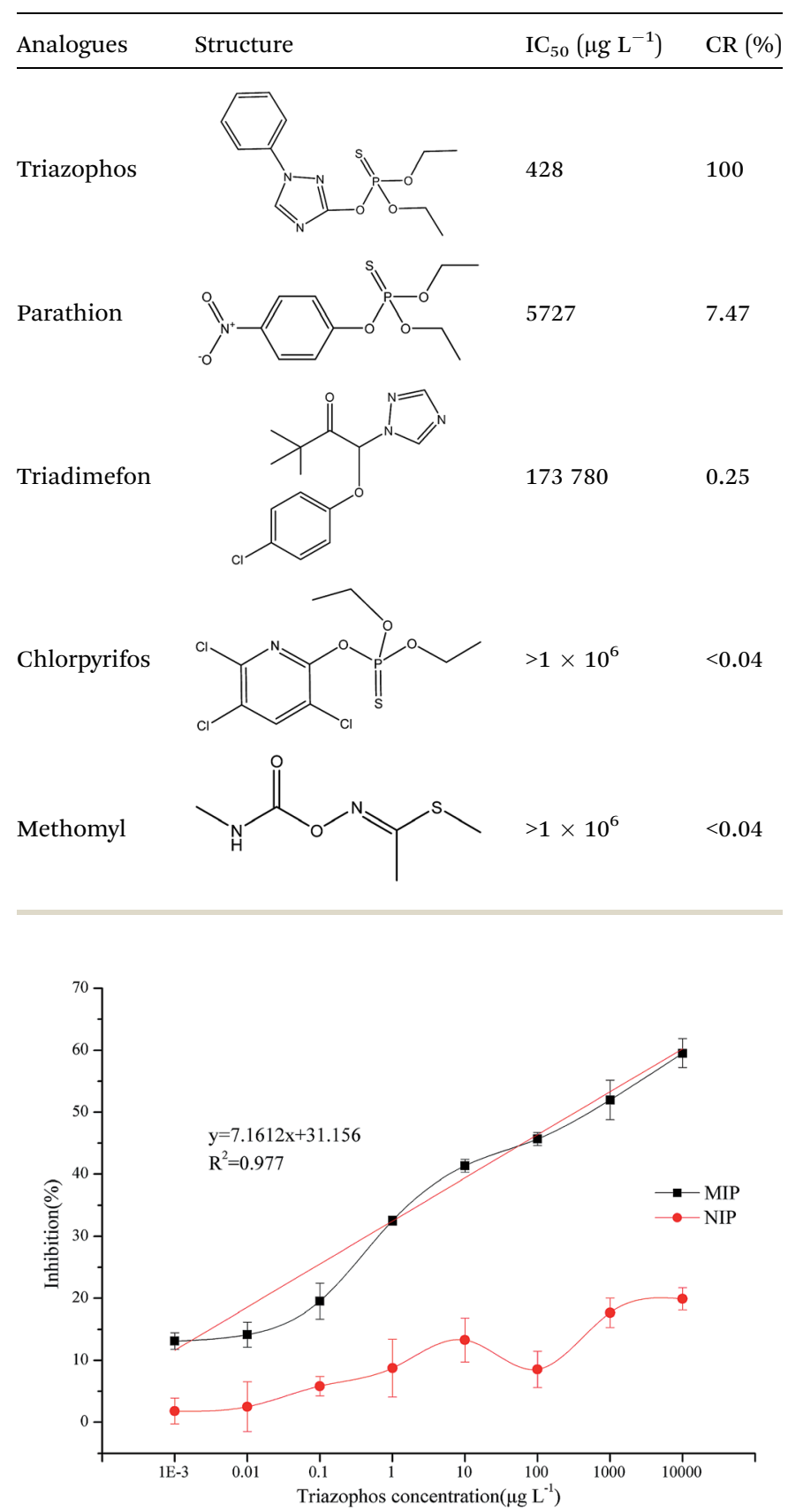

Fig. 10 Triazophos BELISA standard curves using the novel imprinted film and the non-imprinted film as the antibody.

Table 2 Analysis results of spiked triazophos in cabbage and apple samples by BELISA and HPLC-MS/MS methods

\begin{tabular}{llll}
\hline Sample & $\begin{array}{l}\text { Spiking concentration } \\
\left(\mu \mathrm{kg}^{-1}\right)\end{array}$ & $\begin{array}{l}\text { Recoveries/RSD (\%) } \\
\text { BELISA }\end{array}$ & HPLC-MS/MS \\
\hline Cabbage & 10 & $72.0 / 15.6$ & $85.3 / 15.0$ \\
& 50 & $115.1 / 17.4$ & $100.9 / 13.6$ \\
Apple & 500 & $119.8 / 17.5$ & $103.8 / 7.0$ \\
& 10 & $119.7 / 18.9$ & $78.6 / 2.8$ \\
& 50 & $70.5 / 5.2$ & $105.2 / 3.5$ \\
& 500 & $105.9 / 19.7$ & $103.7 / 4.7$
\end{tabular}


Table 3 Comparison between various methods for triazophos detection

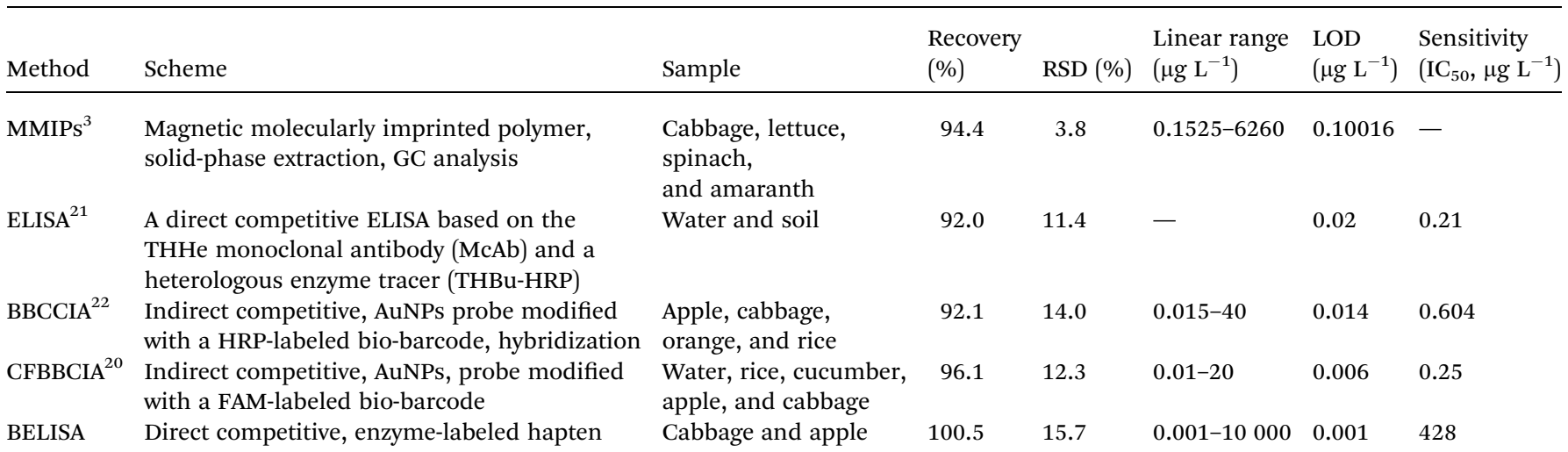

antibody for triazophos at concentrations ranging from $0.001 \mu \mathrm{g} \mathrm{L}{ }^{-1}$ to $10000 \mu \mathrm{g} \mathrm{L}^{-1}$ in $5 \%$ methanol PBS solution are shown in Fig. 10. At the same triazophos concentration, the inhibition rate of the MIP was higher than that of the NIP. When the concentration of triazophos was $10000 \mu \mathrm{g} \mathrm{L}^{-1}$, the inhibition rate of the MIP was $59.5 \%$ and that of the NIP was only $19.9 \%$. Thus, the MIP had better selectivity and adsorption capacity for triazophos than the NIP.

Under optimal conditions, the limit of detection (LOD) and the sensitivity $\left(\mathrm{IC}_{50}\right)$ were $0.001 \mu \mathrm{g} \mathrm{L^{-1 }}$ and $428 \mu \mathrm{g} \mathrm{L}^{-1}$, respectively. The maximum residue level of triazophos in primary products in China is $0.05 \mathrm{mg} \mathrm{kg}{ }^{-1}$. This method can meet the need of sensitivity in trace triazophos determination. Thus, the BELISA method is fully sensitive for detecting triazophos in real samples.

\subsection{Real sample application}

To evaluate the suitability and the applicability of BELISA for the measurement of real samples, cabbage and apple samples spiked at 10,50 , and $500 \mu \mathrm{g} \mathrm{kg}^{-1}$ were investigated (with three replicates for each concentration). The results are shown in Table 2. The recovery of BELISA ranged from 72.0 to $119.8 \%$ in cabbage and from 70.5 to $119.7 \%$ in apple, with the RSD of BELISA ranging from 15.6 to $17.5 \%$ in the cabbage sample and from 5.2 to $19.7 \%$ in the apple sample. The accuracy of BELISA was validated by comparative analysis of the spiked cabbage and apple samples by HPLC-MS/MS with a correlation coefficient $\left(r^{2}\right)$ of 0.9927 . These results indicate that the BELISA method has good accuracy and precision for triazophos detection in agricultural products.

\subsection{Comparison between various methods}

Various methods for triazophos detection are compared in Table 3. We can see that BELISA has a wide linear range, a low LOD and low cost. Besides this, BELISA doesn't need expensive instruments, skilled technicians and a hard prepared antibody. With these advantages, BELISA has potential for application in the quantitative determination of triazophos in agroproducts.

\section{Conclusions}

A sensitive, simple, quick, and direct biomimetic enzyme-linked immunosorbent assay based on a dummy template for the determination of triazophos residues was developed. This method exhibited good performance in the detection of triazophos residues in agro-products after pretreatment. Under optimal conditions, the limit of detection (LOD) and the sensitivity $\left(\mathrm{IC}_{50}\right)$ of the developed BELISA were $0.001 \mu \mathrm{g} \mathrm{L}{ }^{-1}$ and $428 \mu \mathrm{g} \mathrm{L}^{-1}$, respectively. The methodological evaluation of this method showed that it has excellent recovery ranging from 70.5 to $119.8 \%$ and relative standard deviations (RSDs) ranging from 5.2 to $19.7 \%$, when it was used to detect triazophos in cabbage and apple samples. This study also indicated that the developed novel BELISA had particular selectivity toward triazophos using the hapten of triazophos labelled as horseradish peroxidase.

\section{Conflicts of interest}

All of the authors have declared no conflicts of interest.

\section{Acknowledgements}

This work was supported by the National Natural Science Foundation of China (Contact No. 31471654, 31772071,31401590) and the Key Laboratory of Agrifood safety and Quality for MOA (2016KF-10), the Central Public-interest Scientific Institution Basal Research Fund (No. 1610072016009), and the China Agriculture Research System (CARS-05-05A-03).

\section{References}

1 C. Chen, Y. Wang, X. Zhao, Q. Wang and Y. Qian, Ecotoxicology, 2014, 23, 221-228.

2 W. J. Gui, S. T. Wang, Y. R. Guo and G. N. Zhu, Anal. Biochem., 2008, 377, 202-208.

3 H. Li, T. Xie, L. Ye, Y. Wang and C. Xie, Microchim. Acta, 2017, 184, 1011-1019.

4 J. Xiong and B. Hu, J. Chromatogr. A, 2008, 1193, 7-18.

5 Z. Huang, Y. Li, B. Chen and S. Yao, J. Chromatogr. B: Anal. Technol. Biomed. Life Sci., 2007, 853, 154-162. 
6 R. Romero-Gonzalez, A. Garrido Frenich and J. L. Martinez Vidal, Talanta, 2008, 76, 211-225.

7 Y. R. Guo, S. Y. Liu, W. J. Gui and G. N. Zhu, Anal. Biochem., 2009, 389, 32-39.

8 C. Ramansuri, R. Boro, Y. Nangia, S. Gandhi, P. Sharma, N. Wangoo, K. Rajesh and G. Shekhawat, TrAC, Trends Anal. Chem., 2009, 28, 29-39.

9 S. Wang, Z. Xu, G. Fang, Y. Zhang, B. Liu and H. Zhu, J. Agric. Food Chem., 2009, 57, 4528-4534.

10 K. Mosbach, Trends Biochem. Sci., 1994, 19, 9.

11 X. Kan, Y. Zhao, Z. Geng, A. Zhilin Wang and J. J. Zhu, J. Phys. Chem. C, 2008, 112, 4849-4854.

12 L. Li, Z. Lin, A. Peng, H. Zhong, X. Chen and Z. Huang, Food Chem., 2017, 229, 403-408.

13 Q. Sun, L. Xu, Y. Ma, X. Qiao and Z. Xu, J. Sci. Food Agric., 2014, 94, 102-108.

14 C. Shi, X. Liu, L. Song, X. Qiao and Z. Xu, Food Anal. Method, 2015, 8, 2496-2503.
15 W. Lian, S. Liu, L. Wang and H. Liu, Biosens. Bioelectron., 2015, 73, 214-220.

16 L. Cenci, C. Piotto, P. Bettotti and B. Maria, Talanta, 2018, 178, 772-779.

17 D. Zhao, X. Qiao, Z. Xu, R. Xu and Z. Yan, J. Immunoassay Immunochem., 2013, 34, 16-29.

18 J. Pan, X. Zou, X. Wang, W. Guan, Y. Yan and J. Han, Chem. Eng. J., 2010, 162, 910-918.

19 W. J. Gui, R. Y. Jin, Z. L. Chen, J. L. Cheng and G. N. Zhu, Anal. Biochem., 2006, 357, 9-14.

20 C. Zhang, P. Du, Z. Jiang, M. Jin, G. Chen, X. Cao, X. Cui, Y. Zhang, R. Li, A. M. Abd El-Aty and J. Wang, Anal. Chim. Acta, 2018, 999, 123-131.

21 C. Liang, R. Jin, W. Gui and G. Zhu, Environ. Sci. Technol., 2007, 41, 6783.

22 P. Du, M. Jin, G. Chen, C. Zhang, X. Cui, Y. Zhang, Y. Zhang, P. Zou, Z. Jiang, X. Cao, Y. She, F. Jin and J. Wang, Microchim. Acta, 2017, 184, 3705-3712. 